\title{
Refractory cervical esophagogastric anastomotic strictures: Management and outcomes
}

\author{
Samantha J. Davis, BS, ${ }^{a}$ Lili Zhao, $\mathrm{PhD},{ }^{\mathrm{b}}$ Andrew C. Chang, $\mathrm{MD},{ }^{\mathrm{c}}$ and Mark B. Orringer, $\mathrm{MD}^{\mathrm{c}}$
}

\begin{abstract}
Objective: For recalcitrant cervical esophagogastric anastomotic strictures after transhiatal esophagectomy, a protocol of self-dilatation was developed at the University of Michigan Medical Center, as previously described. This study was undertaken to determine the outcomes of this treatment.
\end{abstract}

\begin{abstract}
Methods: Self-dilatation was required in 158 (7.6\%) of 2075 patients with cervical esophagogastric anastomotic strictures after transhiatal esophagectomy. An esophageal-specific survey evaluated the frequency and duration of dilatation, swallowing function, and satisfaction with treatment. The relationship among anastomotic leak, subsequent stricture, and the need for self-dilatation was assessed. A validated survey tool, the Short Form 36-item, version 2, was used to assess quality of life.

Results: At the time of this study, 78 of 158 patients were alive; 34 (43\%) participated in the esophageal-specific survey. Median duration of self-dilatation was 10 years. The majority were satisfied with their ability to eat. No adverse events were reported. All patients said they would use self-dilatation therapy again under similar circumstances. Of these patients, $20(59 \%)$ responded to the Short Form 36-item, version 2. Compared with the general population, $55 \%$ and $70 \%$ of participants scored at or above the norm for physical health and mental health status, respectively. Patients who required self-dilatation were twice as likely to have a history of cervical esophagogastric anastomotic leak as those who did not require this therapy $(P=.0002)$.

Conclusions: Refractory cervical esophagogastric anastomotic strictures are best managed initially with frequent outpatient dilatations, then transitioning to self-dilatation. Home use of Maloney dilators is a safe, well-tolerated, convenient, and cost-effective way to maintain comfortable swallowing. The effectiveness of self-dilatation therapy is reflected in this cohort's good quality of life and level of functioning. (J Thorac Cardiovasc Surg 2011;141:444-8)
\end{abstract}

Most cases of dysphagia after a transhiatal esophagectomy (THE) and cervical esophagogastric anastomosis (CEGA) respond to an occasional esophageal dilatation in the outpatient setting. For more recalcitrant CEGA strictures, a standardized therapeutic protocol has been developed that consists of instruction in and implementation of selfdilatation with a Maloney dilator, as previously described. ${ }^{1}$

Self-dilatation allows patients to maintain comfortable swallowing without the need for repeated trips to the doctor. In addition, the price of a bougie ranges between $\$ 100$ and $\$ 300$, which is a fraction of the cost of multiple visits to a thoracic surgeon or gastroenterologist for dilatations. During the

From the University of Michigan Medical School, ${ }^{\text {a }}$ University of Michigan Comprehensive Cancer Center, ${ }^{\mathrm{b}}$ and University of Michigan Medical Center, ${ }^{\mathrm{c}}$ Section of Thoracic Surgery, Ann Arbor, Mich.

This study was funded by the University of Michigan Section of Thoracic Surgery. Disclosures: Authors have nothing to disclose with regard to commercial support.

Read at the 36th Annual Meeting of The Western Thoracic Surgical Association, Ojai, California, June 23-26, 2010.

Received for publication June 13, 2010; revisions received Sept 23, 2010; accepted for publication Oct 10, 2010; available ahead of print Dec 16, 2010.

Address for reprints: Samantha J. Davis, BS, University of Michigan Medical Center, Department of Surgery, Section of Thoracic Surgery, 2120 Taubman Center, Box 0344, 1500 East Medical Center Drive, Ann Arbor, MI $48109-5344$ (E-mail: sjdmed@umich.edu).

0022-5223/\$36.00

Copyright (c) 2011 by The American Association for Thoracic Surgery doi:10.1016/j.jtcvs.2010.10.011 past 3 decades, many patients with refractory strictures have been managed with this protocol at the University of Michigan Medical Center. However, the efficacy of this program has not been evaluated systematically. This study was undertaken to more objectively define the outcomes of this treatment.

\section{MATERIALS AND METHODS}

This study was approved by the University of Michigan Institutional Review Board.

\section{Patient Selection}

An esophageal resection database of patients treated at the University of Michigan between 1976 and 2009 was reviewed. From this cohort, 2075 patients who underwent THE and CEGA were identified. Billing records were used to identify 158 patients who had been issued dilators for home use. The demographics of this group were compared with the larger cohort of patients who had undergone THE and CEGA (Table 1). The Social Security Death Index and electronic medical records were reviewed to evaluate survival status. Eligible candidates were contacted by mail and asked to return a postcard if interested in taking part in the surveys. Patients who elected to participate were contacted by phone to conduct the esophagealspecific survey (ESS) at 1 time point and were later called to follow up with the Short Form 36-item, version 2 (SF-36v2) survey.

\section{Esophageal-Specific Survey}

A survey was developed to characterize home use of the Maloney dilator and focus on quality of life (QOL) issues related to swallowing. The ESS 


\section{Abbreviations and Acronyms}

CEGA = cervical esophagogastric anastomosis

ESS $=$ Esophageal-Specific Survey

$\mathrm{QOL}=$ quality of life

SF-36v2 = Short Form 36-item, version 2

THE $=$ transhiatal esophagectomy was composed of questions that can be separated into 4 categories: dilator use, dysphagia, patient satisfaction, and insurance coverage for the dilator. Questions about dilator use include whether or not the patients were still using self-dilatation therapy, the frequency with which they were passing the dilator, whether or not someone assisted them (eg, friend, significant other), and if they were no longer passing the dilator, why they had stopped. They were asked about difficulty swallowing and whether or not they could eat what they wanted. Satisfaction with their ability to eat was rated on a scale of 1 to 5 , with 1 being very dissatisfied, 3 being neutral, and 5 being very satisfied. Patients were asked whether or not they would use selfdilatation again given similar circumstances and if their insurance provider covered the cost of the dilator (Figure 1).

\section{Quality of Life Survey}

The SF-36v2 questionnaire was used to assess overall QOL. The SF-36v2 is a well-described QOL measurement tool that has been validated in multiple patient populations, including patients who have undergone esophagectomy. ${ }^{2-4}$ Patients were asked 36 questions about their perceived physical and mental health status. Physical health is subdivided into physical functioning, role-physical, bodily pain, and general health components. These components are summarized by the physical component summary score. Mental health is subdivided into social functioning, roleemotional, vitality, and mental health, which are summarized by the mental component summary score (Table 2).

\section{Statistical Analysis}

Anastomotic leak rates, chemotherapy/radiation therapy, and other demographic variables were compared using a chi-square test. Mean ages at diagnosis were compared using an independent-samples $t$ test. Median duration of self-dilatation was calculated using the Kaplan-Meier method. One-sample $t$ test was used to compare the sample means of SF-36 scores with the corresponding healthy population means. All statistical analyses were performed using SPSS or PASW Statistics software (SPSS Inc, Chicago, IL).

\section{RESULTS}

Of 2075 patients who underwent THE and CEGA between 1976 and 2009, 8\% (158) were taught self- dilatation therapy at some point in the course of their follow-up care. Most demographic characteristics of these patients did not differ significantly from those of patients who underwent THE and CEGA but did not require selfdilatation therapy. However, the rate of anastomotic leak and the use of neoadjuvant chemotherapy or radiation therapy were significantly higher in the self-dilatation cohort (Table 1). The Maloney dilator size most commonly used was $46 \mathrm{~F}$, because this size most often provides normal or near normal swallowing in adults. Of these patients, $21.5 \%$ had a history of a postoperative CEGA leak. In contrast, only $12 \%$ of patients who underwent THE who did not require self-dilatation therapy had a history of CEGA leak $(P=.0002)$. When this study was initiated, 78 of 158 patients $(49 \%)$ were alive, and 34 patients $(43 \%)$ participated in the ESS. The mean age at the time self-dilatation therapy was initiated was 58 years (range 40-80 years). The mean duration of follow-up since surgery was 118 months. The most common indications for esophagectomy in this cohort were adenocarcinoma (22), Barrett's mucosa with high-grade dysplasia (5), and squamous cell carcinoma (3). Other indications included achalasia, recurrent hiatal hernia, and caustic injury (4). Fourteen patients $(41 \%)$ had a history of neoadjuvant chemotherapy or radiation.

The median duration of self-dilatation therapy was approximately 10 years (116 months). The median durations for the 25th and 75th percentiles were 19 and 60 months, respectively. This result excludes data from 2 patients who could not recall when they stopped using self-dilatation. At the time the ESS was conducted, 18 patients $(53 \%)$ were still passing their dilator with an average frequency of once every 64 days. Fourteen patients stopped using their dilator because it was no longer needed, and 2 patients stopped at a physician's request because of a perceived potential for harm. Although 22 patients $(65 \%)$ reported some dysphagia, $29(85 \%)$ were satisfied or very satisfied with their overall ability to eat. The frequency of dysphagia was difficult for most patients to quantify, and it was usually reported as occurring only with consumption of particular foods (ie, bread and meat). There were no reported adverse events from self-dilatation, and all patients surveyed indicated they would use self-dilatation therapy again under

TABLE 1. Demographic variables

\begin{tabular}{lccc}
\hline Study population characteristic & Self-dilatation $(\mathbf{n}=\mathbf{1 5 8})$ & No self-dilatation $(\mathbf{n}=\mathbf{1 9 1 7})$ & $\boldsymbol{P}$ value \\
\hline Mean age & $59.4 \pm 11.8$ y & $61.6 \pm 12.7$ y &. $\mathbf{0 4}$ \\
Male gender & $73.4 \%(116)$ & $76.6 \%(1468)$ & .37 \\
Caucasian race & $96.2 \%(152)$ & $94.9 \%(1819)$ & .47 \\
Malignancy & $74.7 \%(118)$ & $77.8 \%(1492)$ & .36 \\
Anastomotic leak & $21.5 \%(34)$ & $11.5 \%(221)$ & $<.001$ \\
Neoadjuvant chemotherapy or RT & $57.6 \%(68)$ & $42.4 \%(633)$ & .001 \\
Postoperative chemotherapy or RT & $14.4 \%(17)$ & $15.0 \%(224)$ & .86 \\
\hline
\end{tabular}

$R T$, Radiation therapy. 


\begin{tabular}{|c|c|}
\hline ESS Question & Response \\
\hline Are you still using your dilator? & Yes/No \\
\hline - If yes, how often? & Write-in \\
\hline - If no, when \& why did you stop? & Write-in \\
\hline $\begin{array}{l}\text { If no, how often were you passing the dilator when you } \\
\text { stopped? }\end{array}$ & Write-in \\
\hline Have you had any problems related to your self-dilatation therapy? & Yes/No \\
\hline Did anyone assist you? & Yes/No \\
\hline Did you have to be issued $>1$ size? & Yes/No \\
\hline Any difficulty swallowing currently? & Yes/No \\
\hline - $\quad$ If yes, how often? & Rarely - Occasionally - Often - Daily \\
\hline Does food sometimes "stick"? & Yes/No \\
\hline - If yes, how often? & Rarely - Occasionally - Often - Daily \\
\hline Do you currently have any acid regurgitation/heartburn? & Yes/No \\
\hline Do you sleep with your head elevated? & Yes/No \\
\hline Are you able to eat what you want? & Yes/No \\
\hline - If no, please explain. & Write-in \\
\hline How satisfied are you with your ability to eat? & $\begin{array}{c}\text { Very Satisfied - Satisfied - Neutral - } \\
\text { Dissatisfied - Very Dissatisfied }\end{array}$ \\
\hline Are you pleased with the results of self-dilatation therapy? & Yes/No \\
\hline Are you better now than before you started self-dilatations? & Yes/No \\
\hline Under similar circumstances, would you use this therapy again? & Yes/No \\
\hline Did insurance pay for the dilator? & Yes/No \\
\hline Did you pay for any part of the cost of the dilator out-of-pocket? & Yes/No \\
\hline - If yes, what portion? & Less than $50 \%$ - 50\% - More than $50 \%$ - All \\
\hline
\end{tabular}

FIGURE 1. The ESS, which assesses dilatation frequency, patient satisfaction, dysphagia, and insurance coverage for self-dilatation therapy. ESS, Esophageal-Specific Survey.

similar circumstances. Health insurance providers covered at least some of the cost of the dilator in two thirds of cases (23/34).

Of the 34 patients who responded to the ESS, the majority $(59 \%)$ completed the SF-36v2 at a later date. Compared with a normal, healthy population, $55 \%$ of participants scored at or above the mean for physical health status (Figure 2). The physical component summary score encompasses evaluation of physical functioning, bodily pain, rolephysical, and general health. For mental health status, 70\% of responders scored at or above the mean for healthy controls (Figure 3). The mental component summary assesses for vitality, social functioning, role-emotional, and mental health. There were no significant differences in mental com- ponent summary, role-emotional, or mental health mean scores for our patient population compared with the general healthy population (Table 2). Most notable physical limitations reported were caused by disease states unrelated to their esophagectomy or use of self-dilatation therapy.

\section{DISCUSSION}

A CEGA stricture is a relatively common cause of dysphagia after THE, although cervical dysphagia of varying degrees may certainly occur in the absence of an identifiable fibrotic stenosis. There are several methods of esophageal dilatation, including the use of mercury- or tungstenweighted bougies (eg, Maloney dilators), balloon dilators, or fluoroscopic and wire-guided bougies. Blind passage of 
TABLE 2. Quality of life scores: Results of the Short Form 36-item, version 2 survey

\begin{tabular}{|c|c|c|}
\hline SF-36v2 components & $\begin{array}{l}\text { Study population } \\
\text { mean scores }\end{array}$ & $\begin{array}{l}\text { Healthy population } \\
\text { mean scores }\end{array}$ \\
\hline Physical component summary & 45.4 & $55.3^{*}$ \\
\hline Physical functioning & 44.5 & $54.8^{*}$ \\
\hline Role-physical & 46.0 & $54.4^{*}$ \\
\hline Bodily pain & 46.4 & $55.6^{*}$ \\
\hline General health & 49.1 & $55.4^{*}$ \\
\hline Mental component summary & 50.3 & 53.0 \\
\hline Vitality & 49.4 & $54.6^{*}$ \\
\hline Social functioning & 47.9 & $53.8^{*}$ \\
\hline Role-emotional & 47.3 & 53.5 \\
\hline Mental health & 50.0 & 53.4 \\
\hline
\end{tabular}

a Maloney dilator is the simplest and most convenient of these methods, because it can be performed without the use of sedation, the need for postoperative recovery, or exposure to radiation. When refractory strictures that require frequent dilatations develop in patients, safety and ease of use are more important than ever. Home use of Maloney bougienage offers a method of managing strictures that is more convenient yet.

When patients report any degree of dysphagia after construction of a CEGA, they undergo an anastomotic dilatation with Maloney esophageal bougies passed without the use of sedation, generally using $36 \mathrm{~F}, 40 \mathrm{~F}$, and $46 \mathrm{~F}$ dilators.

\section{QOL of Patients Using Self-Dilatation Therapy for CEGA Stricture: \\ SF-36v2 Physical Component Summary}

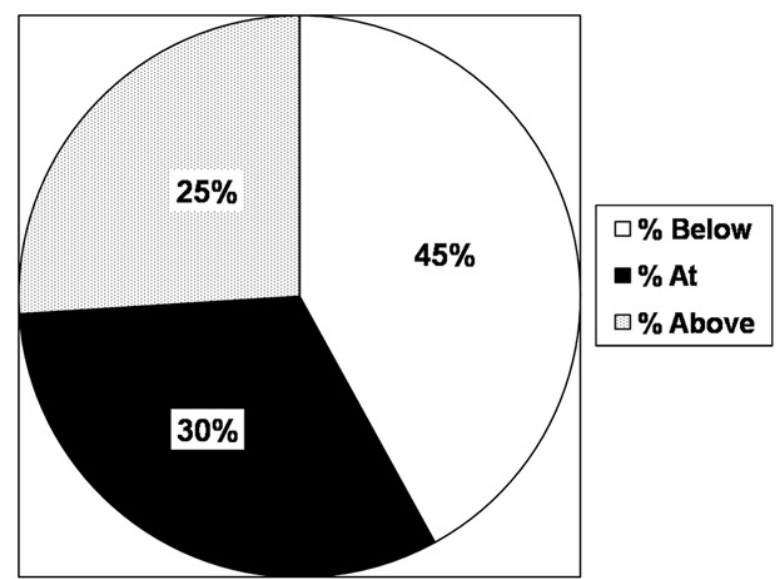

FIGURE 2. Percentage of patients with physical component summary scores above, at, or below the general healthy population mean score. The physical component summary encompasses evaluation of physical functioning, bodily pain, role-physical, and general health. $Q O L$, Quality of life; CEGA, cervical esophagogastric anastomosis; $S F-36 v 2$, Short Form 36-item, version 2.

\section{QOL of Patients Using Self-Dilatation Therapy for CEGA Stricture: \\ SF-36v2 Mental Component Summary}

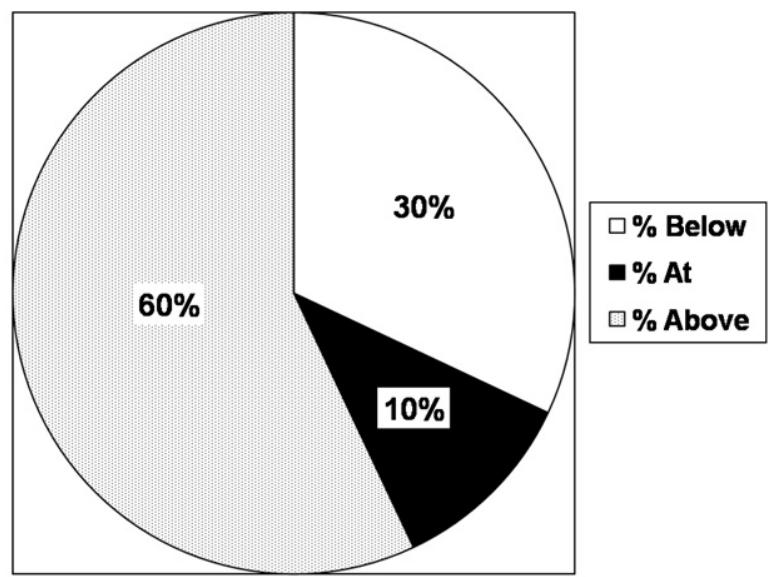

FIGURE 3. Percentage of patients with mental component summary scores above, at, or below the general healthy population mean score. The mental component summary encompasses evaluation of vitality, social functioning, role-emotional, and mental health. $Q O L$, Quality of life; $C E G A$, cervical esophagogastric anastomosis; $S F-36 v 2$, Short Form 36item, version 2 .

If there is no resistance to the passage of the dilators, patients are instructed to return for a follow-up dilatation if their dysphagia recurs. If there is resistance encountered with passage of the $46 \mathrm{~F}$ dilator, or if dysphagia recurs within several weeks of the dilatation, a stricture is presumed to be present on clinical grounds. If a $28 \mathrm{~F}$ dilator cannot be passed blindly through the anastomosis, endoscopic wire-guided dilatation is performed, and the patient returns to the clinic for follow-up Maloney dilatations within 1 week.

When multiple clinic visits for dilatations are required, or when the interval of relief between dilatations is only days or weeks, the stricture is deemed refractory, and self-dilatation is considered. In patients presenting with anastomotic strictures 9 to 12 months or more after an esophagectomy for cancer, the possibility of a malignant stricture must be considered, and endoscopy with brushings for cytology and biopsy should be performed before initiating dilatation therapy.

After determining that a patient is an appropriate candidate for self-dilatation, he or she undergoes biweekly instructional sessions over 2 to 3 weeks with a caregiver present. When the patient has demonstrated competency in performing self-dilatation, a $46 \mathrm{~F}$ Maloney dilator is issued for home use. The patient is instructed to pass the dilator daily for the first week, every other day for the second week, and then at increasingly longer intervals until the longest duration between dilatations without recurrence of dysphagia is determined. 
A recent meta-analysis found that all reported dilatation techniques had comparable safety profiles. This study suggested an algorithm for management of refractory strictures in which self-dilatation was a last resort and an alternative to surgery. ${ }^{5}$ The reluctance to prescribe athome dilatation seems to be largely due to fear regarding patient discomfort and risk of perforation in an unsupervised setting. However, neoplastic or inflammatory distal esophageal stenoses, which may be perforated with Maloney dilatation, ${ }^{6}$ contrast considerably with fibrotic CEGA strictures, which have substantially more structural integrity. Our data suggest that patients prefer self-dilatation, with $85 \%$ being overall satisfied with their ability to eat and all indicating that they would use self-dilatation again if faced with similar circumstances. Revision of an esophageal anastomotic stricture may result in additional anastomotic scarring and recurrent dysphagia and is not, therefore, our recommended approach. In fact, anastomotic revision has only been used in 5 of 2075 patients who underwent THE and CEGA.

\section{CONCLUSIONS}

Deciding who will benefit from self-dilatation and when it should be used can be difficult. Among our 2075 patients, a CEGA leak developed in $11.5 \%$ in the postoperative period (Table 1). In recent years, this rate has decreased because of the use of our stapled anastomotic technique. ${ }^{7} \mathrm{Al}-$ though this complication is easily managed by opening the neck incision at the bedside, it seems to double the likelihood that a refractory stricture will develop later. It has long been our practice to pass up to a $46 \mathrm{~F}$ Maloney dilator before discharge in any patient experiencing a postoperative CEGA leak after THE, which serves both to prevent early anastomotic narrowing and to familiarize the patient with the procedure. Thoracic surgeons should anticipate that patients with a CEGA leak will likely require dilatations at some point, and they should consider discussing selfdilatation with these patients early on. In addition, the majority $(62 \%)$ of patients surveyed required assistance from a caregiver to pass the dilator, at least initially. Patients who have an unstable social situation or difficulty comprehending the dilatation teaching may not be good candidates for this type of therapeutic intervention. The results of our study show that for properly selected candidates, there should be no hesitancy in initiating self-dilatation therapy because of reservations regarding its safety or any perceived negative effects on QOL. Ready access to a full range of sizes of Maloney esophageal dilators and an established process for issuing them for home use should be part of the esophageal surgeon's armamentarium.

\section{References}

1. Chang AC, Orringer MB. Management of the cervical esophagogastric anastomotic stricture. Semin Thorac Cardiovasc Surg. 2007;19:66-71.

2. Chang LC, Oelschlager BK, Quiroga E, et al. Long-term outcome of esophagectomy for high-grade dysplasia or cancer found during surveillance for Barrett's esophagus. J Gastrointest Surg. 2006;10:341-6.

3. Young MM, Deschamps C, Allen MS, et al. Esophageal reconstruction for benign disease: self-assessment of functional outcome and quality of life. Ann Thorac Surg. 2000;70:1799-802.

4. Schembre D, Arai A, Levy S, Farrell-Ross M, Low D. Quality of life after esophagectomy and endoscopic therapy for Barrett's esophagus with dysplasia. Dis Esophagus. 2010;23:458-64. Epub 2010 Jan 26.

5. Siersema PD, de Wijkerslooth LR. Dilation of refractory benign esophageal strictures. Gastrointest Endosc. 2009;70:1000-12.

6. Hernandez LV, Jacobson JW, Harris MS. Comparison among the perforation rates of Maloney, balloon, and savary dilation of esophageal strictures. Gastrointest Endosc. 2000;51:460-2.

7. Orringer MB, Marshall B, Chang AC, et al. Two thousand transhiatal esophagectomies: changing trends, lessons learned. Ann Surg. 2007;246:363-74. 